$052009405200 . h r f$

HUTP-94/A010, hep-th/9405200

\title{
Fusion, mass, and representation theory of the Yangian algebra
}

\author{
Tomoki Nakanishi ${ }^{\dagger}$ \\ nakanisi@string.harvard.edu \\ Lyman Laboratory of Physics \\ Harvard University \\ Cambridge, MA 02138
}

\begin{abstract}
Based on the formulation of Drinfel'd, Chari, and Pressley, a technique to analyze the structure of tensor products of the Yangian algebra representations is presented. We then apply the results to the $S$-matrix theory of the $G \otimes G$-invariant nonlinear $\sigma$-model ( $G$-principal chiral model) by Ogievetsky, Reshetikhin, and Wiegmann. We show how the physical data such as mass formula, fusion angle, and the spins of integrals of motion can be extracted from the Yangian highest weight representations.
\end{abstract}

$05 / 94$

$\dagger$ On leave from Department of Mathematics, Nagoya University, Nagoya, Japan 464 


\section{Introduction}

The Yangian $Y(g)[1]$ is a Hopf algebra associated to a simple Lie algebra $g$.

The structure of the Yangian first appeared in the study of the spectrum of the transfer matrices of a series of integrable spin chains [2-5] in the algebraic Bethe ansatz method (see [6] for a concise introduction), in the study of the elastic S-matrices of two-dimensional relativistic field theory [7-11], and in the system with non-local conserved currents [1215]. These works already exhibited a rich structure and characteristic properties of the Yangian representations. Several representation theoretical ideas, such as $R$-matrix, fusion procedure, were already in [2]. More recently the Yangian also arose in a long-range interacting spin chain $[16,17]$.

At the same time the representation theory of the Yangian has been developed [18-22]. In particular the notion of highest weight representations, parallel to those of simple Lie algebras, was studied in [20-22].

It may therefore be appropriate and useful to describe some of the results in the above mentioned works by using highest weight representations. In this paper we focus on the $S$-matrix theory of the $G \otimes G$-invariant nonlinear $\sigma$-model ( $G$-principal chiral model) in $[9,11]$. It turns out that the highest weight representations are more than a useful language.

They are directly related to the basis of the $S$-matrix theory. Our approach is close to [14] in spirit, but with more emphasis on $Y(g)$ representation theoretical aspects. Some of the issues studied in this paper have been also addressed in [23].

In section 2 we prepare basic facts on Yangian highest weight representations. In section 3 we present a technique to study tensor products of the fundamental representations, including some new results. This provides the proof of the fusion contents of the fundamental representations used in [24]. In section 4 we extend the fundamental representations under the extension of $Y(g)$ by the two-dimensional Poincaré algebra [14]. Applications to an $S$-matrix model are given. First we reproduce the mass spectrum of the $G \otimes G$-invariant nonlinear $\sigma$-model in [9] from the fusion data of $Y(g)$. This is a generalization of the idea of [25] for $Y\left(A_{n}\right)$ to all $Y(g)$. We then show the triangle relation of the fusing angles, and prove that all the bound state poles are indeed in the physical strip. Finally the selection rule for the spins of integrals of motion is derived from the consistency of the fusion data. 


\section{Yangian}

Let $g$ be a simple Lie algebra with an invariant bilinear form $($,$) , and let \left\{I_{p}\right\}$ be an orthonormal basis of $g$. Then, the Yangian $Y(g)$ is the Hopf algebra generated by elements $x, J(x),(x \in g)$ with the relations

$$
\begin{aligned}
& J(a x+b y)=a J(x)+b J(y), \quad a, b \in \mathbf{C}, \quad[x, J(y)]=J([x, y]) \\
& {[J(x), J([y, z])]+[J(y), J([z, x])]+[J(z), J([x, y])]} \\
& =\sum_{p, q, r}\left(\left[x, I_{p}\right],\left[\left[y, I_{q}\right],\left[z, I_{r}\right]\right]\right)\left\{I_{p}, I_{q}, I_{r}\right\} \\
& {[[J(x), J(y)],[z, J(w)]]+[[J(z), J(w)],[x, J(y)]]} \\
& =\sum_{p, q, r}\left(\left(\left[x, I_{p}\right],\left[\left[y, I_{q}\right],\left[[z, w], I_{r}\right]\right]\right)+\left(\left[z, I_{p}\right],\left[\left[w, I_{q}\right],\left[[x, y], I_{r}\right]\right]\right)\right)\left\{I_{p}, I_{q}, J\left(I_{r}\right)\right\}
\end{aligned}
$$

where $\left\{x_{1}, x_{2}, x_{3}\right\}=\frac{1}{24} \sum_{\sigma} x_{\sigma(1)} x_{\sigma(2)} x_{\sigma(3)}$ and the sum is over all the permutations $\sigma$ of $\{1,2,3\}$.

The algebra $Y(g)$ is also realized as an algebra generated by elements $x_{i k}^{ \pm}, h_{i k},(i=$ $\left.1, \ldots, r=\operatorname{rank} g, k \in \mathbf{Z}_{\geq 0}\right)$ with the relations

$$
\begin{gathered}
{\left[h_{i k}, h_{j l}\right]=0, \quad\left[h_{i 0}, x_{j l}^{ \pm}\right]= \pm\left(\alpha_{i}, \alpha_{j}\right) x_{j l}^{ \pm}, \quad\left[x_{i k}^{+}, x_{j l}^{-}\right]=\delta_{i j} h_{i k+l}} \\
{\left[h_{i k+1}, x_{j l}^{ \pm}\right]-\left[h_{i k}, x_{j l+1}^{ \pm}\right]= \pm \frac{1}{2}\left(\alpha_{i}, \alpha_{j}\right)\left(h_{i k} x_{j l}^{ \pm}+x_{j l}^{ \pm} h_{i k}\right)} \\
{\left[x_{i k+1}^{ \pm}, x_{j l}^{ \pm}\right]-\left[x_{i k}^{ \pm}, x_{j l+1}^{ \pm}\right]= \pm \frac{1}{2}\left(\alpha_{i}, \alpha_{j}\right)\left(x_{i k}^{ \pm} x_{j l}^{ \pm}+x_{j l}^{ \pm} x_{i k}^{ \pm}\right)} \\
\sum_{\sigma}\left[x_{i k_{\sigma(1)}}^{ \pm},\left[x_{i k_{\sigma(2)}}^{ \pm}, \ldots,\left[x_{i k_{\sigma\left(1-A_{i j}\right)}^{ \pm}}^{ \pm}, x_{j l}^{ \pm}\right] \ldots\right]\right]=0 \text { for } i \neq j .
\end{gathered}
$$

where $A_{i j}$ is the Cartan matrix of $g$. See [1,20-22] for details. The correspondence from the first to the second realization is given by

$$
\begin{aligned}
h_{i} & \mapsto h_{i 0}, \quad x_{i}^{ \pm} \mapsto x_{i 0}^{ \pm}, \\
J\left(h_{i}\right) & \mapsto h_{i 1}+\frac{1}{4} \sum_{\alpha>0}\left(\alpha, \alpha_{i}\right)\left(x_{\alpha 0}^{+} x_{\alpha 0}^{-}+x_{\alpha 0}^{-} x_{\alpha 0}^{+}\right)-\frac{1}{2} h_{i 0}^{2}, \\
J\left(x_{i}^{ \pm}\right) & \mapsto x_{i 1}^{ \pm} \pm \frac{1}{4} \sum_{\alpha>0}\left(\left[x_{i 0}^{ \pm}, x_{\alpha 0}^{ \pm}\right] x_{\alpha 0}^{\mp}+x_{\alpha 0}^{\mp}\left[x_{i 0}^{ \pm}, x_{\alpha 0}^{ \pm}\right]\right)-\frac{1}{4}\left(x_{i 0}^{ \pm} h_{i 0}+h_{i 0} x_{i 0}^{ \pm}\right),
\end{aligned}
$$

where $x_{\alpha 0}^{ \pm}$are the images of root vectors $x_{\alpha}^{ \pm} \in g,\left(x_{\alpha}^{+}, x_{\alpha}^{-}\right)=1$ of weights $\pm \alpha$ through the above correspondence. 
The advantage of the first realization is that the comultiplication becomes simple, i.e., for $x \in g$,

$$
\Delta(x)=x \otimes 1+1 \otimes x, \quad \Delta(J(x))=J(x) \otimes 1+1 \otimes J(x)+\frac{1}{2}[x \otimes 1, \Omega],
$$

where $\Omega=\sum_{p} I_{p} \otimes I_{p}$. $\Omega$ is related to the second Casimir element of $g, \Omega_{2}=\sum_{p} I_{p} I_{p}$, as

$$
\Omega=\frac{1}{2}\left(\Delta\left(\Omega_{2}\right)-\Omega_{2} \otimes 1-1 \otimes \Omega_{2}\right)
$$

The advantage of the second one is that it admits the Poincaré-Birkhoff-Witt decomposition $Y(g)=Y^{-} H Y^{+}$, where $Y^{ \pm}$and $H$ are subalgebras generated by $x_{i k}^{ \pm}$and $h_{i k}$. Then it is possible to define the notion of a highest weight representation with a highest weight vector $v$ satisfying

$$
x_{i k}^{+} \cdot v=0, \quad h_{i k} \cdot v=d_{i k} v, \quad d_{i k} \in \mathbf{C} .
$$

Let $\tau_{i}=\left(\alpha_{i}, \alpha_{i}\right) / 2$ for each simple root $\alpha_{i}$. There is a fundamental theorem about highest weight representations.

Theorem [20]. 1). Every irreducible finite dimensional representation of $Y(g)$ is highest weight.

2). An irreducible highest weight representation with highest weight $\left\{d_{i k}\right\}$ is finite dimensional if and only if there exist monic polynomials $P_{i}(u)(i=1, \ldots, r)$ such that

$$
\frac{P_{i}\left(u+\tau_{i}\right)}{P_{i}(u)}=1+\sum_{k=0}^{\infty} d_{i k} u^{-k-1}
$$

We call $P_{i}(u)$ 's the Drinfel'd polynomials. Then $d_{i 0}=\tau_{i} \operatorname{deg} P_{i}$ is the $g$-highest weight of a $Y(g)$-highest weight vector. Thus Drinfel'd polynomials are a natural, but a quite non-trivial generalization of the notion of an integral weight of $g$.

In this paper we focus on the irreducible representations such that $\operatorname{deg} P_{i}=\delta_{i a}$ for some $a$. Such representations are called the fundamental representations, and studied in [22]. From now on we choose the normalization of the inner product such that $(\alpha, \alpha)=2$ for any long root $\alpha$. Then the second Casimir of the adjoint representation is equal to $2 g^{\vee}$, where $g^{\vee}$ is the dual coxeter number of $g$. Let $W_{a}(b)(b \in \mathbf{C})$ denote the fundamental representation with the Drinfel'd polynomials

$$
P_{a}(u)=u-b-\frac{1}{2} \tau_{i}+\frac{1}{4} g^{\vee}, \quad P_{i}(u)=1 \quad \text { for } i \neq a
$$




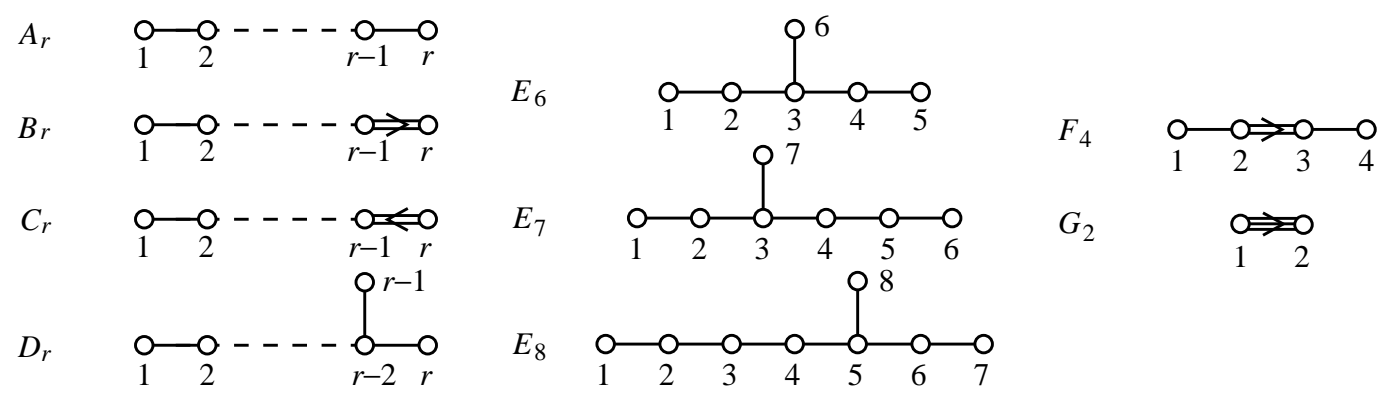

Figure 1. The numbering of the simple roots of the simple Lie algebras.

and let $v_{a}$ be a highest weight vector of $W_{a}(b)$. Then we have

$$
\begin{aligned}
& h_{i k} \cdot v_{a}=\left(b+\frac{1}{2} \tau_{i}-\frac{1}{4} g^{\vee}\right)^{k} h_{i 0} \cdot v_{a}, \\
& x_{i k}^{-} \cdot v_{a}=\left(b+\frac{1}{2} \tau_{i}-\frac{1}{4} g^{\vee}\right)^{k} x_{i 0}^{-} \cdot v_{a} .
\end{aligned}
$$

The first equality is a direct consequence of the definition. The second one follows from the fact that $\left(x_{a k}^{-}-\left(b+\tau_{a} / 2-g^{\vee} / 4\right)^{k} x_{a 0}^{-}\right) \cdot v_{a}$ is a singular vector due to $(2.9 \mathrm{a})$.

Define the action of $x$ and $J(x)$ on $W_{a}(b)$ by the correspondence (2.3). Then from (2.9a) and $(2.9 \mathrm{~b})$ we get (c.f. Lemma 3.4 of $[22])$

$$
J(x) \cdot v_{a}=b x \cdot v_{a}
$$

for all $x=x_{i}^{ \pm}, h_{i}$. To derive this, we have used the formula,

$$
\sum_{\alpha>0}\left(\alpha, \alpha_{i}\right) h_{\alpha}=g^{\vee} h_{i}, \quad \sum_{\alpha>0}\left[x_{\alpha}^{+},\left[x_{i}^{-}, x_{\alpha}^{-}\right]\right]=\left(\left(\alpha_{i}, \alpha_{i}\right)-g^{\vee}\right) x_{i}^{-} .
$$

A simplification of the action of $J(x)$ like (2.10) does not occur for a given vector in $W_{a}(b)$ in general except for $Y\left(A_{r}\right)$ [1]. Nevertheless the action like (2.10) is true in some cases: For instance, if there is a vector $v \in W_{a}(b)$ and $x \in\left\{x_{i}^{ \pm}, h_{i}\right\}$ such that $x \cdot v=x_{i_{1}}^{-} \cdots x_{i_{n}}^{-} \cdot v_{a} \neq 0$ but $x_{i_{\sigma(1)}}^{-} \cdots x_{i_{\sigma(n)}}^{-} \cdot v_{a}=0$ for any permutation $\sigma \neq 1$, then $J(x) \cdot v=b x \cdot v$ due to (2.1a) and (2.10). We use this remark extensively in the next section.

With the above information we can study the structure of the tensor products among

the fundamental representations to some extent. Eq. (2.10) is the key formula, because it gives a bridge between the two realizations of $Y(g)$ in the analysis of highest weight representations.

\section{Tensor product and fusion procedure}

In this section we present a way to study structures of tensor products of fundamental representations. We write the irreducible $g$-module with the highest weight $\Lambda$ as $V_{\Lambda}$, and the $i$-th fundamental weight as $\Lambda_{i}\left(\Lambda_{0}=0\right)$. We fix the numbering of the simple roots as in Fig.1. 


\subsection{Multiplicity free case}

We first consider the case when the decomposition of a tensor product as a $g$-module is multiplicity free (i.e., the dimension of the space of the $g$-highest weight vectors of a given weight is at most one). To make our argument concrete, we use an example in $g=F_{4}$. As a $g$-module the $Y\left(F_{4}\right)$-module $W_{4}(b)$ is isomorphic to $V_{\Lambda_{4}}$, and $J(x)$ acts on $W_{4}(b)$ as $b x$ for any $x \in g[1]$.

Below we shall determine the $Y(g)$-module structure of the tensor product

$$
W_{4}\left(b^{\prime}\right) \otimes W_{4}(b)
$$

We do this in two steps: Step 1). Find all the $Y(g)$-highest weight vectors. Step 2). If there is a $Y(g)$-highest weight vector, determines the Drinfel'd polynomials of the submodule generated by it.

Step 1). Since any $Y(g)$-highest weight vector is $g$-highest, we start by decomposing $W_{4}\left(b^{\prime}\right) \otimes W_{4}(b)$ as a $g$-module to

$$
V_{\Lambda_{4}} \oplus V_{\Lambda_{3}} \oplus V_{2 \Lambda_{4}} \oplus V_{\Lambda_{1}} \oplus V_{\Lambda_{0}}
$$

Let $u_{4}$ and $v_{4}$ be $Y(g)$-highest weight vectors of $W_{4}\left(b^{\prime}\right)$ and $W_{4}(b)$. An explicit form of a $g$-highest weight vector $w_{a}$ of each component in (3.2) is given in Table 1 . It is trivial that $w_{2.4}$ is $Y(g)$-highest. Our main question is whether the other $g$-highest weight vectors are $Y(g)$-highest or not. Since the decomposition (3.2) is multiplicity free, we only need to check the condition

$$
J\left(x_{i}^{+}\right) \cdot w_{a}=0
$$

For each $\Lambda_{a}(a=0,1,3,4)$, we assign the weight $\imath\left(\Lambda_{a}\right)$ such that $\left(x_{i}^{+} \otimes 1\right) \cdot w_{a} \in V_{\imath\left(\Lambda_{a}\right)}$ for all $i$. They are summarized in the following diagram:

$$
\Lambda_{4} \stackrel{\imath}{\longrightarrow} \Lambda_{3} \stackrel{\imath}{\longrightarrow} 2 \Lambda_{4} \stackrel{\imath}{\longleftarrow} \Lambda_{1} \stackrel{\imath}{\longleftarrow} \Lambda_{0}
$$

This graph is essentially the tensor product graph in [23,26-28], a convenient way to keep track of the submodule structure. Because $J(x)$ acts by $b x$ on any vector in $W_{4}(b)$, we have

$$
\left(J\left(x_{i}^{+}\right) \otimes 1\right) \cdot w_{a}=b^{\prime}\left(x_{i}^{+} \otimes 1\right) \cdot w_{a}, \quad\left(1 \otimes J\left(x_{i}^{+}\right)\right) \cdot w_{a}=b\left(1 \otimes x_{i}^{+}\right) \cdot w_{a}
$$


Table 1. Explicit forms of $g$-highest weights in the decomposition (3.2). Here, [34] $\otimes[234]$ is a shorthand for $x_{3}^{-} x_{4}^{-} \cdot u_{4} \otimes x_{2}^{-} x_{3}^{-} x_{4}^{-} \cdot v_{4}$, etc.

\begin{tabular}{|c|c|}
\hline $\mathrm{e}$ & ghest weight vector \\
\hline$V_{2 \Lambda_{4}}$ & $w_{2 \cdot 4}=\mathbf{1} \otimes \mathbf{1}\left(\equiv u_{4} \otimes v_{4}\right)$ \\
\hline$V_{\Lambda_{3}}$ & $w_{3}=[4] \otimes \mathbf{1}-\mathbf{1} \otimes[4]$ \\
\hline$V_{\Lambda_{1}}$ & $\begin{aligned} w_{1}= & {[43234] \otimes \mathbf{1}-[3234] \otimes[4]+[234] \otimes[34]-[34] \otimes[234] } \\
& +[4] \otimes[3234]-\mathbf{1} \otimes[43234]\end{aligned}$ \\
\hline$V_{\Lambda_{4}}$ & $\begin{aligned} w_{4}= & (2 / 3)[43231234] \otimes \mathbf{1}-(1 / 3)[34231234] \otimes \mathbf{1}-[3231234] \otimes[4] \\
& +[231234] \otimes[34]-[31234] \otimes[234]+[1234] \otimes[3234] \\
& +[3234] \otimes[1234]-[234] \otimes[31234]+[34] \otimes[231234] \\
& -[4] \otimes[3231234]-(1 / 3) \mathbf{1} \otimes[34231234]+(2 / 3) \mathbf{1} \otimes[43231234]\end{aligned}$ \\
\hline
\end{tabular}

While (3.5) may not hold in general, there are many situations where (3.5) remains true even if the action of $Y(g)$ is non-trivial as explained for (2.10). The examples (3.14)-(3.17) discussed later are indeed such cases except for the last two cases in (3.17).

Since $w_{a}$ is $g$-highest, we have

$$
\left(x_{i}^{+} \otimes 1\right) \cdot w_{a}=-\left(1 \otimes x_{i}^{+}\right) \cdot w_{a}\left(\equiv y_{i a} \in V_{\imath\left(\Lambda_{a}\right)}\right) .
$$

Using (2.4), (2.5), (3.5), and (3.6), we get

$$
J\left(x_{i}^{+}\right) \cdot w_{a}=\left[b^{\prime}-b+\frac{1}{4}\left(c_{2}\left(\Lambda_{a}\right)-c_{2}\left(\imath\left(\Lambda_{a}\right)\right)\right)\right] y_{i a},
$$

where $c_{2}(\Lambda)$ is the second Casimir of $V_{\Lambda}$. From (3.7) we see that $w_{a}$ is $Y(g)$-highest if and only if ${ }^{\dagger}$

$$
b^{\prime}-b=\frac{1}{4}\left(c_{2}\left(\imath\left(\Lambda_{a}\right)\right)-c_{2}\left(\Lambda_{a}\right)\right) .
$$

The relevant values of the second Casimir are $c_{2}\left(\Lambda_{1}\right)=18, c_{2}\left(\Lambda_{3}\right)=24, c_{2}\left(\Lambda_{4}\right)=12$, and $c_{2}\left(2 \Lambda_{4}\right)=26$ (c.f. [29]).

Step 2). Our next task is to determine the Drinfel'd polynomials of the irreducible submodule generated by $w_{a}$ when $b^{\prime}-b$ takes the value of (3.8). We give two independent ways to do it.

The first way is to calculate the eigenvalue of $J\left(h_{a}\right)$. Since the case $a=0$ is trivial, we concentrate on the cases $a=1,3,4$. Suppose we have the decomposition

$$
\left(h_{a} \otimes 1\right) \cdot w_{a}=y_{\|}+y_{\perp}, \quad y_{\|} \in V_{\Lambda_{a}}, y_{\perp} \in V_{\imath\left(\Lambda_{a}\right)}
$$

$\dagger$ A similar analysis shows that a $g$-lowest weight vector of $V_{\Lambda_{a}}$ in (3.2) is $Y(g)$-lowest if and only if $b^{\prime}-b=\left(c_{2}\left(\imath^{-1}\left(\Lambda_{a}\right)\right)-c_{2}\left(\Lambda_{a}\right)\right) / 4$. 
Let $\eta$ be the number defined by

$$
y_{\|}=\eta h_{a} \cdot w_{a} .
$$

Then a similar calculation to (3.7) shows that

$$
J\left(h_{a}\right) \cdot w_{a}=\left(b+\frac{\eta}{4}\left(c_{2}\left(\imath\left(\Lambda_{a}\right)\right)-c_{2}\left(\Lambda_{a}\right)\right)\right) h_{a} \cdot w_{a} .
$$

(2.10) and (3.11) mean that the submodule generated by $w_{a}$ is $W_{a}\left(b+\eta\left(c_{2}\left(\imath\left(\Lambda_{a}\right)\right)-\right.\right.$ $\left.c_{2}\left(\Lambda_{a}\right)\right) / 4$ ). In our example a direct calculation using the explicit form of $w_{a}$ in Table 1 shows that $\eta=1 / 2$ for $a=1,3$ and 4 .

The second way is due to [25] and more efficient in this example. Let $\Theta=\sum_{p} I_{p} J\left(I_{p}\right)$. Then

$$
\Delta(\Theta)=\Theta \otimes 1+1 \otimes \Theta+\sum_{p}\left(J\left(I_{p}\right) \otimes I_{p}+I_{p} \otimes J\left(I_{p}\right)\right) .
$$

Using (3.12), we have

$$
\Delta(\Theta) \cdot w_{a}=\left(b+\frac{1}{2}\left(b^{\prime}-b\right)\right) c_{2}\left(\Lambda_{a}\right) w_{a} .
$$

This again means that the submodule generated by $w_{a}$ is $W_{a}\left(b+\frac{1}{2}\left(c_{2}\left(\imath\left(\Lambda_{a}\right)\right)-c_{2}\left(\Lambda_{a}\right)\right) / 4\right)$. This ends the description of Step 2.

Summarizing the results of Steps 1 and 2, we obtain the following homomorphisms of $Y(g)$-modules, or the (injective) fusions:

$$
\begin{array}{r}
W_{3}(b) \hookrightarrow W_{4}\left(b+\frac{1}{4}\right) \otimes W_{4}\left(b-\frac{1}{4}\right), \quad W_{1}(b) \hookrightarrow W_{4}(b+1) \otimes W_{4}(b-1), \\
W_{4}(b) \hookrightarrow W_{4}\left(b+\frac{3}{2}\right) \otimes W_{4}\left(b-\frac{3}{2}\right), \quad \mathbf{C} \hookrightarrow W_{4}\left(b+\frac{9}{4}\right) \otimes W_{4}\left(b-\frac{9}{4}\right) .
\end{array}
$$

A hooked arrow $\hookrightarrow$ indicates that it is injective. In the same way, we get

$$
W_{2}(b) \hookrightarrow W_{3}\left(b+\frac{1}{4}\right) \otimes W_{4}\left(b-\frac{1}{2}\right) \hookrightarrow W_{4}\left(b+\frac{1}{2}\right) \otimes W_{4}(b) \otimes W_{4}\left(b-\frac{1}{2}\right) .
$$

For $Y\left(E_{7}\right)$ we get the following fusions, which produce all the fundamental representations from the minimal representation $W_{6}(b)$ :

$$
\begin{aligned}
& W_{6-a}(b) \hookrightarrow W_{7-a}\left(b+\frac{1}{2}\right) \otimes W_{6}\left(b-\frac{a}{2}\right), \quad \text { for } a=1,2,3, \\
& W_{1}(b) \hookrightarrow W_{6}\left(b+\frac{5}{2}\right) \otimes W_{6}\left(b-\frac{5}{2}\right), \quad \mathbf{C} \hookrightarrow W_{6}\left(b+\frac{9}{2}\right) \otimes W_{6}\left(b-\frac{9}{2}\right), \\
& W_{2}(b) \hookrightarrow W_{1}\left(b+\frac{1}{2}\right) \otimes W_{1}\left(b-\frac{1}{2}\right), \quad W_{3}(b) \hookrightarrow W_{2}\left(b+\frac{1}{2}\right) \otimes W_{1}(b-1), \\
& W_{7}(b) \hookrightarrow W_{1}\left(b+\frac{3}{2}\right) \otimes W_{6}(b-2) .
\end{aligned}
$$


For $Y\left(E_{8}\right)$ all the fundamental representations are produced from the minimal representation $W_{1}(b)$ by the fusions,

$$
\begin{aligned}
& W_{a+1}(b) \hookrightarrow W_{a}\left(b+\frac{1}{2}\right) \otimes W_{1}\left(b-\frac{a}{2}\right), \quad \text { for } \quad a=1,2,3,4 \\
& W_{7}(b) \hookrightarrow W_{1}(b+3) \otimes W_{1}(b-3), \quad W_{8}(b) \hookrightarrow W_{1}\left(b+\frac{5}{2}\right) \otimes W_{7}\left(b-\frac{3}{2}\right), \\
& W_{6}(b) \hookrightarrow W_{7}\left(b+\frac{1}{2}\right) \otimes W_{7}\left(b-\frac{1}{2}\right), \quad W_{5}(b) \hookrightarrow W_{6}\left(b+\frac{1}{2}\right) \otimes W_{7}(b-1), \\
& W_{1}(b) \hookrightarrow W_{1}(b+5) \otimes W_{1}(b-5), \quad \mathbf{C} \hookrightarrow W_{1}\left(b+\frac{15}{2}\right) \otimes W_{1}\left(b-\frac{15}{2}\right) .
\end{aligned}
$$

We also used the knowledge of the $R$-matrix from [22] for the embedding of $W_{1}(b)$ and $\mathbf{C}$ in (3.17).

We call a sequence of numbers $\left[b_{1}, \cdots, b_{n}\right]$ a fusion content of $W_{a}(b)$ if there is a fusion $W_{a}(b) \hookrightarrow W_{\min }\left(b+b_{1}\right) \otimes \cdots \otimes W_{\min }\left(b+b_{n}\right)[24] . W_{\min }(b)$ is the minimal representation of $Y(g)$. For instance $W_{\min }(b)=W_{4}(b)$ for $Y\left(F_{4}\right)$. Then the results (3.14) and (3.15) can be written in the form of fusion contents of $W_{1}(b), W_{2}(b)$, and $W_{3}(b)$ as $[1,-1],\left[\frac{1}{2}, 0,-\frac{1}{2}\right]$, and $\left[\frac{1}{4},-\frac{1}{4}\right]$. Either $[0]$ or $\left[\frac{3}{2},-\frac{3}{2}\right]$ is a fusion content of $W_{4}(b)$. In general for a given $W_{a}(b)$ its fusion content is not necessary unique. Fusion contents were used in [24] for the study of the functional relations among the transfer matrices of related lattice models without a proof. Here we provided its proof for $g=F_{4}$ case. All the other fusion contents in [24] have been proved in the same way. In Table 2 we summarize the minimal representations and fusion contents of the fundamental representations for all $Y(g)$. We use them in section 4 .

\subsection{Multiplicity non-free case}

The content of this subsection will not be used later, so that the reader may skip it.

Even when $g$-highest weight vectors in a tensor product have multiplicity greater than one, one can still apply the same technique with a slight modification. Again the problem is to find a $Y(g)$-highest weight vector, which may occur at a certain value of $b^{\prime}-b$, among the $g$-highest weight vectors of a given weight. But this time we need to choose a particular linear combination of them so that it becomes $Y(g)$-highest. Below we show in an example that this procedure is indeed possible if we know the action of $J(x)$ on these $g$-highest weight vectors.

Let $g=G_{2}$. We consider the tensor product $W_{2}\left(b^{\prime}\right) \otimes W_{1}(b)$. As $g$-modules [1]

$$
W_{1}(b) \simeq V_{\Lambda_{1}} \oplus \mathbf{C}, \quad W_{2}\left(b^{\prime}\right) \simeq V_{\Lambda_{2}}
$$


Table 2. Fusion contents of the fundamental representations of $Y(g)$. The second column gives a number $a$ such that $W_{a}(b)$ is the minimal representation. In the third column $a:\left[b_{1}, \ldots, b_{n}\right]$ represents that the $a$-th fundamental representation has a fusion content $\left[b_{1}, \ldots, b_{n}\right] .0$ is a trivial representation. In $g=D_{r}$ and $E_{6}$ two minimal representations are used. $\left[b_{1}, \bar{b}_{2}\right]$ and $\left[\bar{b}_{1}, b_{2}\right]$ in $D_{r}$, for example, mean the fusions in $W_{r}\left(b_{1}\right) \otimes W_{r-1}\left(b_{2}\right)$ and $W_{r-1}\left(b_{1}\right) \otimes W_{r}\left(b_{2}\right)$, respectively. See [24] for related information.

\begin{tabular}{lcll}
\hline$g$ & min & fusion content \\
\hline$A_{r}$ & 1 & $a:\left[\frac{a-1}{2}, \frac{a-3}{2}, \ldots,-\frac{a-1}{2}\right],(1 \leq a \leq r), 0:\left[\frac{r}{2}, \frac{r-2}{2}, \ldots,-\frac{r}{2}\right]$ \\
$B_{r}$ & $r$ & $a:\left[\frac{2 r-2 a-1}{4},-\frac{2 r-2 a-1}{4}\right],(1 \leq a \leq r-1), r:[0], 0:\left[\frac{2 r-1}{4},-\frac{2 r-1}{4}\right]$ \\
$C_{r}$ & 1 & $a:\left[\frac{a-1}{4}, \frac{a-3}{4}, \ldots,-\frac{a-1}{4}\right],(1 \leq a \leq r), 0:\left[\frac{r+1}{4},-\frac{r+1}{4}\right]$ \\
$D_{r}$ & $r-1, r$ & $a:\left[\frac{r-a-1}{2},-\frac{r-a-1}{2}\right],\left[\frac{r-a-1}{2},-\frac{r-a-1}{2}\right],(1 \leq a \leq r-2, r-a$ even $)$ \\
& & $a:\left[\frac{r-a-1}{2},-\frac{\overline{r-a-1}}{2}\right],\left[\frac{r-a-1}{2},-\frac{r-a-1}{2}\right],(1 \leq a \leq r-2, r-a$ odd $)$ \\
& & $r-1:[\overline{0}], r:[0], 0:\left[\frac{r-1}{2},-\frac{r-1}{2}\right],\left[\frac{r-1}{2},-\frac{r-1}{2}\right],(r$ even $)$ \\
& & $0:\left[\frac{r-1}{2},-\frac{r-1}{2}\right],\left[\frac{r-1}{2},-\frac{r-1}{2}\right],(r$ odd $)$ \\
$E_{6}$ & 1,5 & $1:[0],[2,-\overline{2}], 2:\left[\frac{1}{2},-\frac{1}{2}\right], 3:[1,0,-1],[1, \overline{0},-\overline{1}], 4:\left[\frac{1}{2},-\frac{1}{2}\right]$ \\
& & $5:[\overline{0}],[2,-2], 6:\left[\frac{3}{2},-\frac{3}{2}\right],\left[\frac{3}{2},-\frac{3}{2}\right], 0:[\overline{3},-3],[3,-\overline{3}]$ \\
$E_{7}$ & 6 & $1:\left[\frac{5}{2},-\frac{5}{2}\right], 2:[3,2,-2,-3], 3:\left[\frac{7}{2}, \frac{5}{2}, \frac{3}{2},-\frac{3}{2},-\frac{5}{2},-\frac{7}{2}\right],\left[\frac{3}{2}, \frac{1}{2},-\frac{1}{2},-\frac{3}{2}\right]$ \\
& & $4:[1,0,-1], 5:\left[\frac{1}{2},-\frac{1}{2}\right], 6:[0], 7:[4,-1,-2],[2,1,-4], 0:\left[\frac{9}{2},-\frac{9}{2}\right]$ \\
$E_{8}$ & 1 & $1:[0],[5,-5], 2:\left[\frac{1}{2},-\frac{1}{2}\right], 3:[1,0,-1], 4:\left[\frac{3}{2}, \frac{1}{2},-\frac{1}{2},-\frac{3}{2}\right]$ \\
& & $5:[2,1,0,-1,-2],[4,3,2,-2,-3,-4], 6:\left[\frac{7}{2}, \frac{5}{2},-\frac{5}{2},-\frac{7}{2}\right], 7:[3,-3]$ \\
& & $8:\left[\frac{9}{2},-\frac{3}{2},-\frac{5}{2}\right],\left[\frac{5}{2}, \frac{3}{2},-\frac{9}{2}\right], 0:\left[\frac{15}{2},-\frac{15}{2}\right]$ \\
$F_{4}$ & 4 & $1:[1,-1], 2:\left[\frac{1}{2}, 0,-\frac{1}{2}\right], 3:\left[\frac{1}{4},-\frac{1}{4}\right], 4:[0],\left[\frac{3}{2},-\frac{3}{2}\right], 0:\left[\frac{9}{4},-\frac{9}{4}\right]$ \\
$G_{2}$ & 2 & $1:\left[\frac{1}{6},-\frac{1}{6}\right], 2:[0],\left[\frac{2}{3},-\frac{2}{3}\right], 0:[1,-1]$ \\
\hline
\end{tabular}

Thus as a $g$-module,

$$
W_{2}\left(b^{\prime}\right) \otimes W_{1}(b) \simeq V_{\Lambda_{1}+\Lambda_{2}} \oplus V_{2 \Lambda_{2}} \oplus 2 V_{\Lambda_{2}}
$$

Therefore $g$-highest weight vectors with weight $\Lambda_{2}$ is not multiplicity free. We write an $g$-highest weight vectors of $V_{\Lambda_{1}}$ and $V_{\Lambda_{2}}$ in the left hand side of (3.19) as $v_{1}$ and $u_{2}$. Since $V_{\Lambda_{1}}$ is the adjoint representation, we identify $V_{\Lambda_{1}}$ with $g$. Explicitly the correspondence is

$$
\begin{aligned}
& {[2221] \leftrightarrow 6 x_{1}^{+}, \quad[1221] \leftrightarrow-2 x_{2}^{+},} \\
& {[12221] \leftrightarrow-6 h_{1}, \quad[21221] \leftrightarrow 2 h_{2},}
\end{aligned}
$$

and so on (c.f. [30]), where we used the same notation as in Table 1, namely, [2221] = $x_{2}^{-} x_{2}^{-} x_{2}^{-} x_{1}^{-} \cdot v_{1}$, etc. Under our normalization $\left(\alpha_{1}, \alpha_{1}\right)=2$ the action of $J(x)$ on $W_{1}(b)$ is defined by $[1,22]$

$$
J(x) \cdot(y, \lambda)=\left(-\frac{10}{9} \lambda x,(x, y)\right)+b x \cdot(y, \lambda), \quad y \in g, \lambda \in \mathbf{C} .
$$


The action of $J(x)$ on $W_{2}\left(b^{\prime}\right)$ is given by that of $b^{\prime} x$.

Let us choose independent $g$-highest weight vectors, $w_{2}$ and $w_{2}^{\prime}$, of weight $\Lambda_{2}$ in $W_{2}\left(b^{\prime}\right) \otimes W_{1}(b)$ as

$$
\begin{aligned}
& w_{2}= 3[12212] \otimes(\mathbf{1}, 0)-3[2212] \otimes([1], 0)+2[212] \otimes([21], 0)-[12] \otimes([221], 0) \\
&+[2] \otimes([1221], 0)-2 \cdot \mathbf{1} \otimes([21221], 0)+\mathbf{1} \otimes([12221], 0), \\
& w_{2}^{\prime}=\mathbf{1} \otimes(0,1) .
\end{aligned}
$$

Then $\left(x_{i}^{+} \otimes 1\right) \cdot w_{2} \in V_{\Lambda_{1}+\Lambda_{2}} \oplus V_{2 \Lambda_{2}}$ and $\left(x_{i}^{+} \otimes 1\right) \cdot w_{2}^{\prime}=0$. Thus this time the tensor product graph

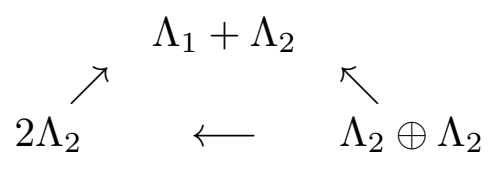

does not immediately tell us whether there exits a $Y(g)$-highest weight vector with weight $\Lambda_{2}$ or not. A direct calculation, using (3.20-22), however shows that for $i=1$ and 2

$$
\begin{array}{r}
J\left(x_{i}^{+}\right) \cdot\left(w_{2}+\alpha w_{2}^{\prime}\right)=\left[b^{\prime}-b-\frac{1}{4}\left(c_{2}\left(\Lambda_{1}+\Lambda_{2}\right)-c_{2}\left(\Lambda_{2}\right)\right)-\alpha\right] y_{i} \\
+\left[b^{\prime}-b-\frac{1}{4}\left(c_{2}\left(2 \Lambda_{2}\right)-c_{2}\left(\Lambda_{2}\right)\right)+\frac{3}{4} \alpha\right] y_{i}^{\prime}
\end{array}
$$

for some nonzero vectors $y_{i} \in V_{\Lambda_{1}+\Lambda_{2}}$ and $y_{i}^{\prime} \in V_{2 \Lambda_{2}}$. Therefore the vector $w_{2}+\alpha w_{2}^{\prime}$ is $Y(g)$-highest if and only if

$$
\begin{aligned}
\alpha & =\frac{1}{7}\left(c_{2}\left(2 \Lambda_{2}\right)-c_{2}\left(\Lambda_{1}+\Lambda_{2}\right)\right), \\
b^{\prime}-b & =\frac{1}{4}\left(\frac{3}{7} c_{2}\left(\Lambda_{1}+\Lambda_{2}\right)+\frac{4}{7} c_{2}\left(2 \Lambda_{2}\right)-c_{2}\left(\Lambda_{2}\right)\right) .
\end{aligned}
$$

Notice that the value $b^{\prime}-b$ in (3.25) is not the difference of two second Casimirs over 4 any more. A similar but a little more nontrivial calculation like (3.9-11) shows

$$
J\left(h_{2}\right) \cdot\left(w_{2}+\alpha w_{2}^{\prime}\right)=\left(b-\frac{\alpha}{4}\right) h_{2} \cdot w_{2}+\left(b^{\prime}+\frac{10}{9 \alpha}\right) h_{2} \cdot \alpha w_{2}^{\prime}
$$

for $\alpha$ and $b^{\prime}-b$ satisfying (3.25). Substituting the values $c_{2}\left(\Lambda_{1}+\Lambda_{2}\right)=14, c_{2}\left(2 \Lambda_{2}\right)=28 / 3$, $c_{2}\left(\Lambda_{1}\right)=8$, and $c_{2}\left(\Lambda_{2}\right)=4$, we have $\alpha=-\frac{2}{3}$ and $b^{\prime}-b=\frac{11}{6}$. Thus we obtain the following fusion

$$
W_{2}(b) \hookrightarrow W_{2}\left(b+\frac{5}{3}\right) \otimes W_{1}\left(b-\frac{1}{6}\right) .
$$




\subsection{Duality}

The existence of a dual (contragradient) representation is guaranteed by the antipode of $Y(g)$. We use a theorem from [22], Proposition 2.17: If $V_{\Lambda_{\bar{a}}}$ is the dual representation of $V_{\Lambda_{a}}$ for $g$, then $W_{\bar{a}}\left(b-\frac{g^{\vee}}{2}\right)$ is dual to $W_{a}(b)$ in the following sense:

i). $\quad \mathbf{C} \hookrightarrow W_{a}(b) \otimes W_{\bar{a}}\left(b-\frac{g^{\vee}}{2}\right)$.

ii). For any finite-dimensional representations $U$ and $V$ of $Y(g)$

$$
\operatorname{Hom}_{Y(g)}\left(U, W_{a}(b) \otimes V\right) \simeq \operatorname{Hom}_{Y(g)}\left(W_{\bar{a}}\left(b-\frac{g^{\vee}}{2}\right) \otimes U, V\right) .
$$

(3.28b), in particular, means if there is a nontrivial homomorphism $U \rightarrow W_{a}(b) \otimes V$, there is also a nontrivial homomorphism $W_{\bar{a}}\left(b-\frac{g^{\vee}}{2}\right) \otimes U \rightarrow V$.

So, if $g \neq A_{r}, D_{r}, E_{6}$, then $W_{a}\left(b-\frac{g^{\vee}}{2}\right)$ is dual to $W_{a}(b)$. For $g=A_{r}, D_{r}, E_{6}$ the diagram automorphism of $g$ is also an automorphism of $Y(g)$. Thus, a fusion

$$
W_{a_{3}}\left(b_{3}\right) \hookrightarrow W_{a_{1}}\left(b_{1}\right) \otimes W_{a_{2}}\left(b_{2}\right)
$$

is equivalent to its conjugate fusion

$$
W_{\bar{a}_{3}}\left(b_{3}\right) \hookrightarrow W_{\bar{a}_{1}}\left(b_{1}\right) \otimes W_{\bar{a}_{2}}\left(b_{2}\right) .
$$

On the other hand, by tensoring $W_{\bar{a}_{3}}\left(b_{3}-\frac{g^{\vee}}{2}\right)$ to (3.29) from the right, then using (3.28a), we have

$$
\mathbf{C} \hookrightarrow W_{a_{1}}\left(b_{1}\right) \otimes W_{a_{2}}\left(b_{2}\right) \otimes W_{a_{3}}\left(\bar{b}_{3}-\frac{g^{\vee}}{2}\right) .
$$

From (3.31) and the remark after (3.28b), we have a nontrivial homomorphism,

$$
W_{\bar{a}_{2}}\left(b_{2}-\frac{g^{\vee}}{2}\right) \otimes W_{\bar{a}_{1}}\left(b_{1}-\frac{g^{\vee}}{2}\right) \rightarrow W_{\bar{a}_{3}}\left(b_{3}-\frac{g^{\vee}}{2}\right) .
$$

Since $W_{\bar{a}_{3}}\left(b_{3}\right)$ is $Y(g)$-irreducible, this homomorphism is surjective by Schur's lemma. Thus (3.29) is also equivalent to the following surjective fusion:

$$
W_{a_{2}}\left(b_{2}\right) \otimes W_{a_{1}}\left(b_{1}\right) \rightarrow W_{a_{3}}\left(b_{3}\right)
$$

\section{Applications to an $S$-matrix model with the $Y(g)$ symmetry}

\subsection{Extension by the Poincaré algebra and mass formula}

We recall [14] that $Y(g)$ defined by (2.1) admits a non-trivial extension by the on-shell two-dimensional Poincaré algebra $\mathcal{P}$,

$$
\left[L, P^{0}\right]=P^{1}, \quad\left[L, P^{1}\right]=P^{0}, \quad\left[P^{0}, P^{1}\right]=0, \quad\left(P^{0}\right)^{2}-\left(P^{1}\right)^{2}=m^{2},
$$


where $L, P^{0}, P^{1}$ are the Lorentz boost, the energy, the momentum operators, and $m$ is a central element. The only non-trivial relation between $Y(g)$ and $\mathcal{P}$ is

$$
[L, J(x)]=\gamma^{-1} x
$$

where $\gamma$ is a coupling constant between the two algebras. Then one can easily check (see Appendix) that (4.2) is compatible with the relations (2.1) and (4.1). The comultiplication for $X \in \mathcal{P}$ is defined as

$$
\Delta(X)=X \otimes 1+1 \otimes X
$$

Let us write this extended Hopf algebra as $P Y(g)$.

Remarkably, like the Virasoro algebra in an affine Lie algebra, the operator $L$ is already built into $Y(g)$. To see this, we use a one-parameter family of automorphisms $T_{b}(b \in \mathbf{C})$ of $Y(g)$ such that [1]

$$
T_{b}: x \mapsto x, \quad T_{b}: J(x) \mapsto J(x)+b x
$$

The pull-back of $W_{a}(0)$ by the map $T_{b}: Y(g) \rightarrow Y(g)$ induces a $Y(g)$-module, which is isomorphic to $W_{a}(b)$ due to Prop. 2.14 in [22]. Thus one can define a representation of $P Y(g)$ on $W_{a}(b)$ as

$$
\begin{aligned}
& J(x)=J(x)_{\text {for } b=0}+b x, \quad L=\frac{1}{\gamma} \frac{\partial}{\partial b}, \\
& P^{0}=m_{a} \cosh \left(\gamma b+d_{a}\right), \quad P^{1}=m_{a} \sinh \left(\gamma b+d_{a}\right) .
\end{aligned}
$$

The constants $m_{a}$ and $d_{a}$ are arbitrary at this moment. This representation is a generalization of the ideas of $[14,25,23]$.

Now, following [25], suppose a quantum field theory possesses the $Y(g)$ symmetry. Furthermore suppose that there are $r(=\operatorname{rank} g)$ particles $a=1, \ldots, r$, and each particle $a$ consists of a multiplet belonging to the fundamental representation $W_{a}(b)$ with the mass $m_{a}>0$. The $G \otimes G$-invariant nonlinear $\sigma$-model is an example [9,11]. Let us assume that a bound state occurs when there is a corresponding, either injective or surjective fusion (we return to this point in section 4.2 again). Then let us see what the results (3.14) and (3.15) in the previous section imply for the $Y\left(F_{4}\right)$ case. The energy and momentum 
Table 3. The dual Coxeter number of $g$.

\begin{tabular}{llllllllll}
\hline$g$ & $A_{r}$ & $B_{r}$ & $C_{r}$ & $D_{r}$ & $E_{6}$ & $E_{7}$ & $E_{8}$ & $F_{4}$ & $G_{2}$
\end{tabular}

dual Coxeter number $r+12 r-1 r+12 r-2 \quad 12 \quad 18 \quad 30 \quad 9 \quad 4$

conservations (4.3) give constraints on $m_{a}$ and $d_{a}$, which are summarized into a set of equations,

$$
\begin{aligned}
m_{4}\left(e^{\gamma}+e^{-\gamma}\right) & =m_{1} e^{ \pm\left(d_{1}-d_{4}\right)}, \\
m_{4}\left(e^{\gamma / 2}+1+e^{-\gamma / 2}\right) & =m_{2} e^{ \pm\left(d_{2}-d_{4}\right)}, \\
m_{4}\left(e^{\gamma / 4}+e^{-\gamma / 4}\right) & =m_{3} e^{ \pm\left(d_{3}-d_{4}\right)}, \\
m_{4}\left(e^{3 \gamma / 2}+e^{-3 \gamma / 2}\right) & =m_{4}, \\
m_{4}\left(e^{9 \gamma / 4}+e^{-9 \gamma / 4}\right) & =0 .
\end{aligned}
$$

In the last equation of (4.6) we required the mass $m_{0}$ of the trivial representation $\mathbf{C}$ to be zero. It is immediate to see that it has a solution only if $d_{a}$ is independent of $a$. From now on we set $d_{a}=0$ without losing generality. Thus the rapidity $\theta_{a}$ of $W_{a}(b)$ is identified with $\gamma b$. Requiring $m_{a}>0,(4.6)$ determines $\gamma$ and $m_{a}$ as

$$
\begin{gathered}
\gamma= \pm \frac{2 \pi i}{9} \bmod 8 \pi i \\
m_{1}=\left(2 \cos \frac{2 \pi}{9}\right) m_{4}, \quad m_{2}=\left(1+2 \cos \frac{\pi}{9}\right) m_{4}, \quad m_{3}=\left(2 \cos \frac{\pi}{18}\right) m_{4} .
\end{gathered}
$$

This agrees with the mass formula in [9], upon correcting the obvious typographical errors therein.

It is straightforward to repeat this calculation for all $Y(g)$. Except for $Y\left(G_{2}\right)$ the requirements $m_{a}>0$ and $m_{0}=0$ discretize the coupling constant $\gamma$ as

$$
\gamma= \pm \frac{2 \pi i}{g^{\vee}} \bmod 4 d \pi i, \quad d= \begin{cases}3 & \text { for } G_{2}, \\ 2 & \text { for } B_{r}, C_{r}, \text { and } F_{4} \\ 1 & \text { otherwize }\end{cases}
$$

The value of the dual Coxeter number $g^{\vee}$ is listed in Table 3. The ratios of the masses $m_{a}$ are unique under (4.8). Let $\left[b_{1}, \cdots, b_{n}\right]$ be a fusion content of $W_{a}(b)$ in Table 2 , and $m_{\min }$ be the mass of the minimal representation $W_{\min }(b)$. Then the mass $m_{a}$ is given in a unified way:

\section{Mass formula.}

$$
m_{a}=m_{\min } \cdot \sum_{j=1}^{n} e^{2 b_{j} \pi i / g^{\vee}}
$$


In the case $Y\left(G_{2}\right)$, besides the above, there is one more positive mass solution,

$$
\gamma= \pm \frac{5 \pi i}{2} \quad \bmod 12 \pi i, \quad m_{1}=\left(2 \cos \frac{5 \pi}{12}\right) m_{2}
$$

It is nothing surprising that (4.9) with the data of the fusion contents in Table 2 reproduces the mass formula in $[9],{ }^{\dagger}$ because the equations in (4.6) are equivalent to the ones used in the bootstrap method $[8,9,11]$. We, however, clarified here how the two-dimensional kinematics is compatible with $Y(g)$.

\subsection{Triangle relation and physical strip condition}

So far we have considered only the positivity condition of mass for the restriction of the coupling constant $\gamma$. In [14] it was mentioned that only the value $\gamma=2 \pi i / g^{\vee}$ ensures the crossing symmetry of the $S$-matrix. (If there is a crossing symmetric $S$-matrix at $\gamma=2 \pi i / g^{\vee}$, however, then there is also one at $\gamma=-2 \pi i / g^{\vee}$.) Below we shall find the values $\gamma= \pm 2 \pi i / g^{\vee}$ using a different argument.

Let $\bar{a}$ denote the antiparticle of $a$. General theory of $S$-matrices tells that if there is a bound state $\bar{a}_{3}$ in the process $a_{1}+a_{2} \rightarrow \bar{a}_{3}$, then the $S$-matrix, as a function of the difference of the rapidities, say, $\theta_{1}-\theta_{2}$, has a pole at a point $i u_{a_{1} a_{2}}^{\bar{a}_{3}}$ with

$$
0<u_{a_{1} a_{2}}^{\bar{a}_{3}}<\pi
$$

We call $u_{a_{1} a_{2}}^{\bar{a}_{3}}$ the fusion angle, and (4.11) the physical strip condition. On the other hand the energy and momentum conservations, like (4.6), give a relation,

$$
m_{a_{3}}^{2}=m_{a_{1}}^{2}+m_{a_{2}}^{2}+2 m_{a_{1}} m_{a_{2}} \cos u_{a_{1} a_{2}}^{\bar{a}_{3}}
$$

among the masses and the fusion angle. Let us further assume that there are also the binding processes $a_{2}+a_{3} \rightarrow \bar{a}_{1}$ and $a_{3}+a_{1} \rightarrow \bar{a}_{2}$. Then using (4.11) and (4.12), we have the triangle relation:

$$
u_{a_{1} a_{2}}^{\bar{a}_{3}}+u_{a_{2} a_{3}}^{\bar{a}_{1}}+u_{a_{3} a_{1}}^{\bar{a}_{2}}=2 \pi .
$$

See $[8,31]$ for details. We shall show that (4.13) follows from the representation theory of $Y(g)$ if and only if we choose $\gamma= \pm 2 \pi i / g^{\vee}$ among the possible values in (4.8) and (4.10).

Below we must use one unproven fact:

$\dagger \quad$ In [9] the mass $m_{3}$ (in their notation) of $E_{6}$ should read as $(\sqrt{3}+1) m_{1}$, and $m_{5}$ and $m_{6}$ of $E_{8}$ should be interchanged. 
Assumption. In our convention of the comultiplication (2.4), there is an injective fusion,

$$
W_{a_{3}}\left(b_{3}\right) \hookrightarrow W_{a_{1}}\left(b_{1}\right) \otimes W_{a_{2}}\left(b_{2}\right),
$$

only if $b_{1}>b_{2}$.

This is supposed to be true in the following reason. In general, if $b_{1}<b_{2}$, then any non-trivial submodule in $W=W_{a_{1}}\left(b_{1}\right) \otimes W_{a_{2}}\left(b_{2}\right)$ has the highest weight $\Lambda_{a_{1}}+\Lambda_{a_{2}}$. In other words $W_{a_{3}}\left(b_{3}\right)$ may be isomorphic to a quotient, but not to a submodule of $W$. Yet we do not know a general proof of the statement at this moment.

Now suppose there is a fusion

$$
W_{\bar{a}_{3}}\left(b_{3}\right) \hookrightarrow W_{a_{1}}\left(b_{1}\right) \otimes W_{a_{2}}\left(b_{2}\right) .
$$

By an argument similar to the one from (3.29) to (3.32), we can show the existence of another fusion

$$
W_{\bar{a}_{1}}\left(b_{1}-\frac{g^{\vee}}{2}\right) \hookrightarrow W_{a_{2}}\left(b_{2}\right) \otimes W_{a_{3}}\left(b_{3}-\frac{g^{\vee}}{2}\right) .
$$

Repeating the procedure once more, we also have

$$
W_{\bar{a}_{2}}\left(b_{2}-\frac{g^{\vee}}{2}\right) \hookrightarrow W_{a_{3}}\left(b_{3}-\frac{g^{\vee}}{2}\right) \otimes W_{a_{1}}\left(b_{1}-g^{\vee}\right) .
$$

The differences of the rapidities in (4.15)-(4.17) are

$$
\theta_{a_{1} a_{2}}^{\bar{a}_{3}}=\gamma\left(b_{1}-b_{2}\right), \quad \theta_{a_{2} a_{3}}^{\bar{a}_{1}}=\gamma\left(b_{2}-b_{3}+\frac{g^{\vee}}{2}\right), \quad \theta_{a_{3} a_{1}}^{\bar{a}_{2}}=\gamma\left(b_{3}-b_{1}+\frac{g^{\vee}}{2}\right) .
$$

Therefore

$$
\theta_{a_{1} a_{2}}^{\bar{a}_{3}}+\theta_{a_{2} a_{3}}^{\bar{a}_{1}}+\theta_{a_{3} a_{1}}^{\bar{a}_{2}}=\gamma g^{\vee} .
$$

So far we have not yet used our assumption on (4.14). As an example, let $g=G_{2}$. From (3.27) we have $\theta_{12}^{\overline{2}}=\theta_{21}^{\overline{2}}=(11 / 6) \gamma$. Also the fusion content of $W_{1}(b)$ in Table 2 gives $\theta_{22}^{\overline{1}}=(1 / 3) \gamma$. Thus $\theta_{12}^{\overline{2}}+\theta_{22}^{\overline{1}}+\theta_{21}^{\overline{2}}=4 \gamma=g^{\vee} \gamma$.

Now let $\gamma=2 \pi i / g^{\vee}$, and let $u_{a_{1} a_{2}}^{\bar{a}_{3}}=-i \theta_{a_{1} a_{2}}^{\bar{a}_{3}}$, etc. Then from the assumption on (4.14) we have $u_{a_{1} a_{2}}^{\bar{a}_{3}}, u_{a_{2} a_{3}}^{\bar{a}_{1}}, u_{a_{3} a_{1}}^{\bar{a}_{2}}>0$, and from (4.19) they satisfies the triangle relation (4.13). Furthermore it is an exercise of elementary geometry to show

$$
u_{a_{1} a_{2}}^{\bar{a}_{3}}, u_{a_{2} a_{3}}^{\bar{a}_{1}}, u_{a_{3} a_{1}}^{\bar{a}_{2}}<\pi
$$

from (4.12) and (4.13). We can repeat the same argument for $\gamma=-2 \pi i / g^{\vee}$ using the duals of (4.15)-(4.17). We conclude that if $\gamma=2 \pi i / g^{\vee}$ (resp. $\gamma=-2 \pi i / g^{\vee}$ ), then any injective (resp. surjective) fusion occurs in the region $0<\operatorname{Im} \theta_{1}-\theta_{2}<\pi$.

Thus $Y(g)$ representation theory guarantees that i) all the fusion angles among fundamental representations are automatically inside the physical strip, ii) if there is a binding process $a_{1}+a_{2} \rightarrow \bar{a}_{3}$, then there are also the others, $a_{2}+a_{3} \rightarrow \bar{a}_{1}$ and $a_{3}+a_{1} \rightarrow \bar{a}_{2}$. 


\subsection{Integrals of motion}

It is commonly believed that in classical and quantum field theories the integrability and the existence of the infinite numbers of integrals of motion (IM) commuting with each other are synonymous. It is an interesting problem to realize the corresponding conserved currents as composite operators of the non-local conserved current in [14].

Suppose we have an IM $I_{s}$ of spin $s$, i.e., $\left[L, I_{s}\right]=s I_{s}$. Then the charge of $I_{s}$ on the particle $a$ with rapidity $\theta_{a}$ is $\omega_{a} e^{s \theta_{a}}$ for some number $\omega_{a}$ We call $\omega_{a}$ the scalar charge. In [31] it was pointed out that the conservation of the $I_{s}$ charge gives a strong constraint among the the spin $s$ and the fusion angles. When $g$ is simply-laced, the mass spectrum of (4.9), thus the fusion angles, are identical to the one in the affine Toda field theory where the situation is well-known [32-34]; an IM with spin $s$ exists if and only if $s$ is an exponent of $g$ modulo the Coxeter number.

We set $\gamma= \pm 2 \pi i / g^{\vee}$ in the rest of the paper. Let us first consider $g=F_{4}$, for example. The conservation of the $I_{s}$ charge under the fusion $W_{4}(b) \hookrightarrow W_{4}\left(b+\frac{3}{2}\right) \otimes W_{4}\left(b-\frac{3}{2}\right)$ in (3.14) gives an consistency equation for $s$,

$$
e^{3 s \gamma / 2}+e^{-3 s \gamma / 2}=2 \cos \frac{s \pi}{3}=1
$$

This excludes the spins $s=0,2,3,4 \bmod 6$. Remembering that the exponents of $F_{4}$ are $1,5,7,11$ and the Coxeter number is 12 , we have already obtained a selection rule:

Selection rule. If an $S$-matrix has the $Y(g)$ symmetry, then an integral of motion with spin $s$ exists only if $s$ is an exponent of $g$ modulo the Coxeter number.

Of course this argument does not necessarily guarantee the existence of an IM at the allowed spins. The systematic absence of IMs at these particular values, however, suggests that IMs really exist at the allowed spins. Below we give the derivation of the above selection rule for all the other algebras. The Coxeter number and the exponents of $g$ are listed in Table 4.

$A_{r}$ : A fusion content of $\mathbf{C}$ is $\left[\frac{r}{2}, \frac{r-2}{2}, \ldots,-\frac{r}{2}\right]$. This leads to a constraint

$$
\sum_{j=0}^{r} e^{2 s j \pi i /(r+1)}=0
$$

Thus $s=0 \bmod r+1$ are excluded. 
Table 4. The Coxeter number and the exponents of $g$.

\begin{tabular}{lcl}
\hline$g$ & Coxeter number & \multicolumn{1}{c}{ exponents } \\
\hline$A_{r}$ & $r+1$ & $1,2, \ldots, r$ \\
$B_{r}$ & $2 r$ & $1,3,5, \ldots, 2 r-1$ \\
$C_{r}$ & $2 r$ & $1,3,5, \ldots, 2 r-1$ \\
$D_{r}$ & $2 r-2$ & $1,3,5, \ldots, 2 r-3, r-1$ \\
$E_{6}$ & 12 & $1,4,5,7,8,11$ \\
$E_{7}$ & 18 & $1,5,7,9,11,13,17$ \\
$E_{8}$ & 30 & $1,7,11,13,17,19,23,29$ \\
$F_{4}$ & 12 & $1,5,7,11$ \\
$G_{2}$ & 6 & 1,5 \\
\hline
\end{tabular}

$B_{r}$ : A fusion content of $\mathbf{C}$ is $\left[\frac{2 r-1}{4},-\frac{2 r-1}{4}\right]$. This leads to a constraint

$$
\cos \frac{s \pi}{2}=0
$$

Thus $s=$ even are excluded.

$C_{r}$ : A fusion content of $\mathbf{C}$ is $\left[\frac{r+1}{4},-\frac{r+1}{4}\right]$. This leads to the same constraint as $(4.23)$. Thus $s=$ even are excluded.

$D_{r}\left(r\right.$ even): A fusion content of $\mathbf{C}$ is $\left[\frac{r-1}{2},-\frac{r-1}{2}\right]$. This leads to the same constraint as (4.23). Thus $s=$ even are excluded.

$D_{r}\left(r\right.$ odd): This and $E_{6}$ cases require two-steps examination. There are two minimal representations $W_{r}(b)$ and $W_{r-1}(b)$, the spin and conjugate spin representations. Let $\omega$ and $\omega^{\prime}$ be the scalar charges of an IM $I_{s}$ on the particles $r$ and $r-1$. For $r-a$ even, there are two fusion contents of $W_{a}(b),\left[\frac{r-a-1}{2},-\frac{r-a-1}{2}\right]$ and $\left[\frac{\overline{r-a-1}}{2},-\frac{\overline{r-a-1}}{2}\right]$. They lead to a consistency equation

$$
\left(\omega-\omega^{\prime}\right) \cos \frac{s(r-a-1) \pi}{2 r-2}=0 .
$$

If $s=r-1 \bmod 2 r-2$, it gives no constraint for $\omega$ and $\omega^{\prime}$. If $s \neq r-1 \bmod 2 r-2$, we have $\omega=\omega^{\prime}$. The fusion content of $\mathbf{C},\left[\frac{r-1}{2},-\frac{\overline{r-1}}{2}\right]$, leads to another constraint

$$
\omega e^{s \pi i / 2}+\omega^{\prime} e^{-s \pi i / 2}=0 .
$$

If $s=r-1 \bmod 2 r-2$, then $\omega=-\omega^{\prime}$ satisfies (4.25). Therefore there is no constraint for $s$. If $s \neq r-1 \bmod 2 r-2$, where $\omega=\omega^{\prime},(4.25)$ excludes $s=$ even $\neq r-1 \bmod 2 r-2$. 
$E_{6}$ : There are two minimal representations $W_{1}(b)$ and $W_{5}(b)$. Let $\omega$ and $\omega^{\prime}$ be the scalar charges of an IM $I_{s}$ on the particles 1 and 5 . The fusion contents, $[2,-2]$ of $W_{5}(b)$ and $[\overline{2},-\overline{2}]$ of $W_{1}(b)$, give equations

$$
\omega^{\prime}=\left(2 \cos \frac{s \pi}{3}\right) \omega, \quad \omega=\left(2 \cos \frac{s \pi}{3}\right) \omega^{\prime},
$$

which exclude $s=0 \bmod 3$, and set $\omega=\omega^{\prime}$ for $s=1,5 \bmod 6$ and $\omega=-\omega^{\prime}$ for $s=2,4$ mod 6. Next compare two fusion contents of $W_{6}(b),\left[\frac{3}{2},-\frac{\overline{3}}{2}\right]$ and $\left[\frac{\overline{3}}{2},-\frac{3}{2}\right]$. This does not give any constraint for $s=1,5 \bmod 6$, but gives a constraint $\sin \frac{s \pi}{4}=0$ for $s=2,4 \bmod$ 6. This excludes $s=2,10 \bmod 12$. Hence we obtain the rule. Alternatively we can get the same result as follows: The fusion contents $[2,-2]$ of $W_{5}(b)$ and $[\overline{1}, \overline{0},-\overline{1}]$ of $W_{3}(b)$ mean another fusion content $[3,-1,2,-2,1,-3]$ of $W_{3}(b)$. Comparing this with $[1,0,-1]$, we have a consistency equation

$$
\cos \frac{s \pi}{2}+\cos \frac{s \pi}{3}=\frac{1}{2} .
$$

$s$ is a solution of (4.27) if and only if it is an exponent of $E_{6} \bmod 12$.

$E_{7}$ : Comparing two fusion contents of $W_{3}(b),\left[\frac{7}{2}, \frac{5}{2}, \frac{3}{2},-\frac{3}{2},-\frac{5}{2},-\frac{7}{2}\right]$ and $\left[\frac{3}{2}, \frac{1}{2},-\frac{1}{2},-\frac{3}{2}\right]$, we have an equation

$$
\cos \frac{7 s \pi}{18}+\cos \frac{5 s \pi}{18}-\cos \frac{s \pi}{18}=0
$$

$s$ is a solution of (4.28) if and only if it is an exponent of $E_{7} \bmod 18$. Thus the other spins are excluded.

$E_{8}$ : Comparing two fusion contents of $W_{5}(b),[4,3,2,-2,-3,-4]$ and $[2,1,0,-1,-2]$, we have an equation

$$
\cos \frac{4 s \pi}{15}+\cos \frac{3 s \pi}{15}-\cos \frac{s \pi}{15}=\frac{1}{2} .
$$

$s$ is a solution of (4.29) if and only if it is an exponent of $E_{8} \bmod 30$. Thus the other spins are excluded.

$G_{2}$ : A fusion content of $W_{2}(b)$ is $\left[\frac{2}{3},-\frac{2}{3}\right]$. This leads to the same constraint as (4.21). Thus $s=0,2,3,4 \bmod 6$ are excluded.

To sum up, the selection rule follows only from the consistency of the fusion data in a remarkably simple way.

Like (4.9), when there is an IM $I_{s}$ of spin $s$, the ratios of the scalar charges of $I_{s}$ are given in terms of a fusion content of $W_{a}(b)$ as

$$
\omega_{a}=\omega_{\min } \cdot \sum_{j=1}^{n} e^{2 s b_{j} \pi i / g^{\vee}} \text { for } g \neq D_{r}, E_{6} .
$$


Here $\omega_{\min }$ is the scalar charge on the particle corresponding to the minimal representation. For $g=D_{r}$ and $E_{6}$, by (4.25) and (4.26), (4.30) is modified as

$$
\omega_{a}=\omega_{\min } \cdot\left(\sum_{j=1}^{n} e^{2 s b_{j} \pi i / g^{\vee}}-(-1)^{s} \sum_{j=1}^{n^{\prime}} e^{2 s b_{j}^{\prime} \pi i / g^{\vee}}\right),
$$

where $\omega_{\min }$ is the scalar charge of the particle $r$ (resp. the particle 1 ) for $D_{r}$ (resp. $E_{6}$ ), and $\left[b_{1}, \ldots, b_{n},{\overline{b^{\prime}}}_{1}, \ldots,{\overline{b^{\prime}}}_{n^{\prime}}\right]$ is a fusion content of $W_{a}(b)$. It is easy to check the property $\omega_{a}=-(-1)^{s} \omega_{\bar{a}}$ from (4.31) [32]. For a simply-laced $g$ the numbers $\omega_{a}(a=1, \ldots, r)$ constitute an eigenvector of the Cartan algebra of $g$ with eigenvalue $2-2 \cos \left(s \pi / g^{\vee}\right)$ as expected from the result in the corresponding affine Toda theory for $g^{(1)}$ [33]. For a nonsimply-laced $g$ those numbers $\omega_{a}$ coincide with the scalar charges of IMs in the classical affine Toda theory for the dual of $g^{(1)}$, which is a twisted affine Lie algebra $[33,35,36]^{\dagger}$.

\section{Conclusion}

We showed how representation theory of $Y(g)$ in $[1,20-22]$ works well in the study of the fusion procedures, the mass spectrum, the triangle relation, and integrals of motion when the $S$-matrix of a massive model has the $Y(g)$ symmetry. We observed that the Yangian perfectly integrates the $S$-matrix theory into itself. This is why the traditional bootstrap approach in $[8,11]$ worked so consistently. Also this reminds us what the affine Lie algebra did for the Wess-Zumino-Witten conformal field theory. This point of view has been already stated in [14]. Technically the key to go beyond the traditional bootstrap and the $R$-matrix method was the identification of a one-particle state with a (fundamental) highest weight representation parametrized by the Drinfel'd polynomials.

Finally it is important to understand any intrinsic relation between the fusion of the Yangian representations and the one described by the Coxeter element [37].

\section{Acknowledgements}

We would like to thank A. Kuniba for pointing out to me the importance of the Drinfel'd polynomials, and thank to him and J. Suzuki for the collaboration which leads to the work presented here. We thank J. Beck, I. Cherednik, S. Cordes, P. Dorey, M.T. Grisaru,

$\dagger$ We thank P. Dorey for making us aware of this point. 
N. MacKay, P. Mathieu, P.B. Wiegmann for interesting discussion and correspondence, M. Bershadsky and C. Vafa for their warm hospitality. This work is supported by JSPS fellowship, NSF grant PHY 92-18167, and Packard fellowship.

\section{Appendix. Compatibility between (2.1) and (4.2)}

The relation (4.2) must be compatible with the relations (2.1) and (4.1). The compatibility with (4.1) is clear.

To check the compatibility with $(2.1)$, we have only to apply $\gamma[L, \cdot]$ to the relations in (2.1) and see the equality. The case (2.1a) is immediate.

i). (2.1b). The action of $\gamma[L, \cdot]$ on the lhs of $(2.1 \mathrm{~b})$ is

$$
\begin{aligned}
& {[x, J([y, z])]+[J(x),[y, z]]+(\operatorname{cyclic} \text { on } x, y, z) } \\
= & 2 J([x,[y, z]])+(\operatorname{cyclic} \text { on } x, y, z)=0 .
\end{aligned}
$$

On the other hand, the action of $\gamma[L, \cdot]$ on the rhs of (2.1b) is zero because there is no $J(x)$ in it.

ii). (2.1c). Using (2.1a) and (2.1b), we have

$$
\begin{aligned}
& \gamma[L,[[J(x), J(y)],[z, J(w)]]] \\
= & {[[x, J(y)],[z, J(w)]]+[[J(x), y],[z, J(w)]]+[[J(x), J(y)],[z, w]] } \\
= & \left.2\left[J([x, y]), J\left(z^{\prime}\right)\right]-\left[\left[J(y), z^{\prime}\right], J(x)\right]-\left[\left[z^{\prime}, J(x)\right], J(y)\right] \quad \quad \quad \text { where } z^{\prime}=[z, w]\right) \\
= & 3\left[J([x, y]), J\left(z^{\prime}\right)\right]+\left\{\left[J(x), J\left(\left[y, z^{\prime}\right]\right)\right]+\left[J(y), J\left(\left[z^{\prime}, x\right]\right)\right]+\left[J\left(z^{\prime}\right), J([x, y])\right]\right\} \\
= & 3[J([x, y]), J([z, w])]+\sum_{p, q, r}\left(\left[x, I_{p}\right],\left[\left[y, I_{q}\right],\left[[z, w], I_{r}\right]\right]\right)\left\{I_{p}, I_{q}, I_{r}\right\} .
\end{aligned}
$$

Thus the action of $\gamma[L, \cdot]$ on the lhs of (2.1c) is

$$
\sum_{p, q, r}\left(\left(\left[x, I_{p}\right],\left[\left[y, I_{q}\right],\left[[z, w], I_{r}\right]\right]\right)+\left(\left[z, I_{p}\right],\left[\left[w, I_{q}\right],\left[[x, y], I_{r}\right]\right]\right)\right)\left\{I_{p}, I_{q}, I_{r}\right\}
$$

which is equal to the action of $\gamma[L, \cdot]$ on the rhs of (2.1c).

These calculations are actually a part of a proof of the fact that (4.4) is an automorphism of $Y(g)$.

\section{References}

[1] V.G. Drinfel'd, Sov. Math. Dokl. 32 (1985) 254; in Proceedings of the International Congress of Mathematicians, Berkeley, (American Mathematical Society, Providence, 1987) 
[2] P.P. Kulish, N.Yu. Reshetikhin and E. K. Sklyanin, Lett. Math. Phys. 5 (1981) 393

[3] P.P. Kulish and E.K. Sklyanin, J. Sov. Math. 19 (1982) 1596; in Lecture Notes in Physics 151 (Springer, Berlin, 1982)

[4] Yu.N. Reshetikhin, Lett. Math. Phys. 7 (1983) 205

[5] A.N. Kirillov and N.Yu. Reshetikhin, Zap. Nauch. Semin. LOMI 160 (1987) 211 [J. Sov. Math. 52 (1990) 3156]

[6] E.K. Sklyanin, hep-th/9211111

[7] B. Berg, M. Karowski, V. Kurak and P. Weiz, Nucl. Phys. B134 (1978) 125

[8] A.B. Zamolodchikov and Al.B. Zamolodchikov, Ann. Phys. 120 (1979) 253

[9] E.I. Ogievetsky and P.B. Wiegmann, Phys. Lett. B168 (1986) 360

[10] E.I. Ogievetsky, J. Phys. G 12 (1986) L105

[11] E.I. Ogievetsky, N.Yu. Reshetikhin and P.B. Wiegmann, Nucl. Phys. B280 [FS 18] (1987) 45

[12] M. Lüscher, Nucl. Phys. B135 (1978) 1

[13] H. de Vega, H. Eichenherr, J.M. Maillet, Nucl. Phys. B240 [FS12] 377

[14] D. Bernard, Commun. Math. Phys. 137 (1991) 191

[15] A. LeClair and F. Simirnov, Int. J. Mod. Phys. A7 (1992) 2997

[16] F.D. Haldane, Z.N.C. Ha, J.C. Talstra, D. Bernard and V. Pasquier, Phys. Rev. Lett. 69 (1992) 2021

[17] D. Belnard, M. Gaudin, F.D.M. Haldane and V. Pasquier, J. Phys. A:Math. Gen. 26 (1993) 5219

[18] V.O. Tarasov, Theore. Math. Phys. 61 (1984) 163; 63 (1985) 175

[19] I. Cherednik, in Proc. of the XVII International Conference on Differential Geometric Methods in Theoretical Physics, Chester, ed. A.I. Solomon, (World Scientific, Singapore, 1989)

[20] V.G. Drinfel'd, Soviet Math. Dokl. 36 (1988) 212

[21] V. Chari and A. Pressley, L'Enseignement Math. 36 (1990) 267

[22] V. Chari and A. Pressley, J. reine angew. Math. 417 (1991) 87

[23] N.J. MacKay, J. Phys. A: Math. Gen. 25 (1992) L1343

[24] A. Kuniba, T. Nakanishi, J. Suzuki, hep-th/9309137, to appear in Int. J. Mod. Phys. A

[25] A.A. Belavin, Phys. Lett. B283 (1992) 67

[26] N.J. MacKay, J. Phys. A: Math. Gen. 24 (1991) 4017

[27] R.B. Zhang, M.D. Gould and A.J. Bracken, Nucl. Phys. B354 (1991) 625

[28] G.W. Delius, M.D. Gould and Y.-Z. Zhang, hep-th/9405030

[29] W.G. McKay and J. Patera, Tables of Dimensions, Indices, and Branching Rules for Representations of Simple Lie Algebras, (Marcel Dekker, New York and Basel, 1981)

[30] W. Fulton and J. Harris, Representation Theory, (Springer-Verlag, New York, 1991)

[31] A.B. Zamolodchikov, Int. J. Mod. Phys. A4 (1989) 4235

[32] V.A. Fateev and A.B. Zamolodchikov, Int. J. Mod. Phys. A5 (1990) 1025

[33] H.W. Braden, E. Corrigan, P.E. Dorey, and R. Sasaki, Nucl. Phys. B338 (1990) 689; B356 (1991) 469

[34] B.L. Feigin and E. Frenkel, hep-th/9310022 and references therein

[35] G.W. Delius, M.T. Grisaru, and D. Zanon, Nucl. Phys. B382 (1992) 365

[36] P. Dorey, Phys. Lett. B312 (1993) 291

[37] P. Dorey, Nucl. Phys. B358 (1991) 654; Nucl. Phys. B374 (1992) 741 